\title{
Streptococcus agalactiae and Chlamydia trachomatis detection in women without symptoms of infection
}

\author{
Magdalena Frej-Mądrzak 1,A-D,F, Agnieszka Jama-Kmiecik1, , , Jolanta Sarowska, , , \\ Dorota Teryks-Wołyniec ${ }^{1, A-C}$, Anna Gryboś2,A,B,F, Marian Grybośs, ${ }^{3, B}$, Irena Choroszy-Król ${ }^{1, A}$ \\ 1 Department of Basic Sciences, Faculty of Health Sciences, Wroclaw Medical University, Poland \\ ${ }^{2}$ Department of Gynaecology and Obstetrics, Faculty of Health Sciences, Wroclaw Medical University, Poland \\ ${ }^{3}$ Faculty of Medical Sciences, Public Higher Medical Professional School in Opole, Poland \\ A - research concept and design; $\mathrm{B}$ - collection and/or assembly of data; $\mathrm{C}$ - data analysis and interpretation; \\ $\mathrm{D}$ - writing the article; $\mathrm{E}$ - critical revision of the article; $\mathrm{F}$ - final approval of the article
}

Address for correspondence

Magdalena Frej-Mądrzak

E-mail: magdalena.frej-madrzak@umed.wroc.pl

\section{Funding sources}

This study was generously supported by grant No. E090.18.021 from the Wroclaw Medical University, Poland.

Conflict of interest

None declared

Received on August 28, 2019

Reviewed on September 7, 2019

Accepted on May 14, 2020

Published online on June 26, 2020

Cite as

Frej-Mądrzak M, Jama-Kmiecik A, Sarowska J, et al.

Streptococcus agalactiae and Chlamydia trachomatis detection in women without symptoms of infection. Adv Clin Exp Med. 2020;29(6):707-713. doi:10.17219/acem/122397

DOI

10.17219/acem/122397

Copyright

Copyright by Author(s)

This is an article distributed under the terms of the

Creative Commons Attribution 3.0 Unported (CC BY 3.0)

(https://creativecommons.org/licenses/by/3.0/)

\begin{abstract}
Background. Chlamydia trachomatis (C. trachomatis) and Streptococcus agalactiae (GBS) may be present in the female cervical canal without any symptoms of infection. Chronic chlamydial infections lead to many serious complications and perinatal infections, while the presence of GBS is a reservoir for infections of newborns or invasive streptococcal infection in adults.
\end{abstract}

Objectives. To examine healthy women for C. trachomatis without symptoms from the reproductive system, assess the frequency of asymptomatic infections, detect GBS in the cervical canal, demonstrate differences in drug susceptibility, and determine the serotype of $S$. agalactiae strains and correlations among the ones present in the cervical canal.

Material and methods. A total of 315 cervical swabs were collected for genetic and microbiological analysis for the presence of $C$. trachomatis and $S$. agalactiae. Latex and diffusion-disk methods were used to determine the serotype and susceptibility of streptococci.

Results. Ten out of 315 women (3.2\%) were C. trachomatis-positive. Using traditional methods of microscopy, culture and serology, 42 strains (13.3\% of the subjects) obtained from patients were identified as $S$. agalactiae and further analyzed. The most common serotypes identified were II (18/42, 42.9\%), V (11/42, 26.2\%) and III (10/42, 23.8\%). The less common serotypes found were VII (2/10, 4.8\%), and Ib (1/10, 2.4\%); no la, IV or VII serotypes were found. All the strains were susceptible to penicillin, while $71.4 \%$ of them were susceptible to erythromycin and $81.0 \%$ were susceptible to clindamycin. Seven isolates (16.7\%) were concomitantly resistant to erythromycin and clindamycin.

Conclusions. Chlamydia trachomatis was confirmed in 3.2\% of the respondents, and GBS was found in 13.3\%, despite a lack of symptoms of infection. The incidence of C. trachomatis infections and GBS colonization in Poland is similar to those in other European countries.

Key words: Chlamydia trachomatis, Streptococcus agalactiae, cervical infection 


\section{Introduction}

Streptococcus agalactiae or Group B Streptococcus (GBS) is a Gram-positive $\beta$ hemolytic coccus in the Streptococcus genus. Streptococcus agalactiae most commonly colonizes the lower gastrointestinal tract, anus and the vaginal environment. Epidemiological data indicates that GBS is found in the vaginal tract of $10-30 \%$ of healthy women who usually do not show any symptoms of inflammation. ${ }^{1-3}$ In non-pregnant women and in men, GBS is becoming an increasingly common cause of invasive diseases, especially in the elderly, in immunocompromised patients or in those with other (particularly chronic) diseases. The combination of bacterial and host factors determines the course of infection. ${ }^{1-3}$ Streptococcus agalactiae causes infection of the skin and subcutaneous tissue, urinary tract, lungs, and endocardium; it also causes group B streptococcal meningitis, which is an important but uncommon manifestation of invasive GBS disease in adults, accounting for up to $4 \%$ of all cases of bacterial meningitis in adults. Most cases of GBS meningitis occur in postpartum women, the elderly or adults with serious underlying diseases. ${ }^{4}$ Symptoms of the disease are generally abrupt, and bacteremia occurs in about $80 \%$ of cases. A distant focus of infection, such as the endometrium or endocarditis, is often identified. The case-mortality rate is high (27-34\%) and closely related to the presence of underlying conditions other than pregnancy. A small but significant proportion of survivors (7\%) suffer from permanent hearing loss. ${ }^{5}$ Streptococcus agalactiae is particularly dangerous for pregnant women, in whom it multiplies intensively in the vaginal environment during pregnancy and poses a real risk to both the mother and the fetus. ${ }^{6}$ Cervical infections in pregnant women are associated with gynecological-obstetrical complications: miscarriage, premature childbirth, fetal membrane rupture, or pelvic inflammatory disease (PID). ${ }^{7}$

Infants can be infected through aspiration of infected amniotic fluid or during childbirth. ${ }^{2,6}$ Group B Streptococcus is associated with invasive disease in newborns. Newborn infections are classified as early when the disease develops in the $1^{\text {st }}$ week of life and manifests as sepsis and pneumonia (so-called early-onset GBS). Late-onset GBS is diagnosed if symptoms appear after the $7^{\text {th }}$ day of life, last until the $3^{\text {rd }}$ month and resemble meningitis. In pregnant women or immediately postpartum, GBS are responsible for inflammation of the urinary tract, fetal membrane and endometrium, for sepsis, and rarely for meningitis. ${ }^{2}$

Chlamydia trachomatis and S. agalactiae may be present in the female cervical canal without any symptoms of infection. Chronic chlamydial infections lead to many serious complications in women and perinatal infections, while the presence of GBS is a reservoir for infections of newborns or invasive streptococcal infection in adults. Chlamydia trachomatis is a bacterium with 3 biotypes responsible for different infections. The $1^{\text {st }}$ one causes pneumonia in mice; the $2^{\text {nd }}$ one, lymphogranuloma venereum (LGV; serotypes L1-L3), is responsible for LGV; and the $3^{\text {rd }}$ one (serotypes $\mathrm{A}-\mathrm{C}$ ) leads to trachoma or urogenital infections, conjunctivitis in adults and children, and pediatric pneumonia (serotypes D-K). Chlamydia trachomatis (especially D-K serotypes) is the most common infection worldwide. ${ }^{8}$

Transmission of C. trachomatis usually takes place through direct mucous membrane contact (vagina, anus) with an infected person during sexual intercourse or oral sex, or immediately after birth through mother's infected cervical canal. The risk of transmission during a single act of vaginal intercourse is estimated to be $10 \%$, and about $55 \%$ for people who have had at least 2 sexual partners in the last 6 months. Partners of people with C. trachomatis infections are very likely to be infected, so it is important to notify and treat them. Despite literature reports on spontaneous clearance of C. trachomatis, it is recommended that appropriate tests be performed in symptomatic and asymptomatic sexually active individuals, and after pathogen identification, to treat infected individuals and their sexual partners from the previous 6 months.

Due to its affinity for the female columnar epithelium, C. trachomatis infects the cervix, urethra and rectum, which leads to cervicitis, and inflammation of the fallopian tubes and pelvic organs. The complications may include infertility, peritoneal tissue inflammation (which occurs throughout the continuity of tissues), the Fitz-Hugh-Curtis syndrome (PID with peritoneal tissue inflammation) and conjunctivitis, most often as an autoinfection. In women, inflammation of the rectum may occur through direct infection and/or PID complications.

In Europe, detected and registered C. trachomatis infections mainly affect heterosexual women (51\%) and men (35\%); perinatal infections amount to $<1 \%$, and the remaining infections appear in homosexual men (10\%) or are unspecified. ${ }^{9}$ However, these values are underestimated due to the small number of reports on such infections. Only 1,628 cases out of over 2 million in Europe were registered in Poland in 2013-2017.9

The developmental lifecycle of C. trachomatis is intracellular and lasts up to $72 \mathrm{~h}$, during which the bacterium occurs as an elementary body (EB), i.e., an infectious form of the bacteria incapable of division, and as a reticular body (RB), which multiplies in a host. Under unfavorable conditions, the bacteria can pass into persistent forms unable to transform into EB, which blocks the cycle and leads to the formation of large atypical forms. Factors that induce the formation of persistent forms in vitro include $\beta$-lactam antibiotics, e.g., penicillin, which block $\mathrm{RB}$ division and prevent further transformation into EBs. Exposure to $\beta$-lactam antibiotics results in the accumulation of large aberrant RBs, so-called penicillin forms. ${ }^{10}$ Moreover, the formation of persistent forms of bacteria is influenced by nutritional factors such as decreased access to basic amino acids and ions, e.g., lowered exogenous 
tryptophan or iron levels, and immunological factors - pro-inflammatory cytokines and interferon $\gamma$ (IFN $\gamma$ ). The interferon blocks RB replication - such large aberrant forms of Chlamydia can be observed in cells with IFNy under in vitro conditions. ${ }^{11}$ Chronic and persistent infections are more likely to cause complications.

In 70-95\% of women, chlamydial infections are asymptomatic. If symptoms do occur, they usually develop 1014 days after sexual contact and most frequently include mucosal cervicitis, later purulent, cervical bleeding, friability, edema, and ulcers. ${ }^{12}$ Furthermore, C. trachomatis infection is accompanied by dysuria, vaginal discharge, post-coital and intermenstrual bleeding, and poorly differentiated abdominal pain or lower abdominal pain. In women, asymptomatic or untreated chlamydial infection can lead to complications such as PID (including endometritis, salpingitis, parametritis, tubo-ovarian abscess, or peritonitis), chronic pelvic pain, infertility, ectopic pregnancy, or fetal membrane rupture. Symptoms suggesting PID include tenderness and pain in the abdomen and lower abdomen - usually bilateral - tenderness and pain during gynecological examinations, acute dyspareunia, abnormal bleeding, as well as abnormal discharge from the vagina or cervix as a result of cervicitis, endometritis or bacterial vaginosis. Moreover, women may develop sexually acquired reactive arthritis (SARA) $(<1 \%) .{ }^{13}$ Adult inclusion conjunctivitis is most commonly associated with urogenital infections (autoinfection) with clinical presentation that includes tearing, conjunctival congestion, photophobia, moderately swollen eyelids, and the presence of mucus. This conjunctivitis does not lead to blindness, although complications such as corneal pannus or ulcer have been observed. ${ }^{14}$

The Polish Gynecological Society recommends annual C. trachomatis screening to pregnant and non-pregnant women $\leq 25$ years (especially before a planned pregnancy). Screening in pregnant women should be performed in the $1^{\text {st }}$ and $3^{\text {rd }}$ trimester of pregnancy (during the first visit). Non-pregnant women older than 25 years should be examined at least once a year - in particular women who engaged in high-risk sexual behavior before a planned pregnancy. Pregnant women $>25$ years should be examined in the $1^{\text {st }}$ trimester (recommended during their first visit), and in the $3^{\text {rd }}$ trimester only if at risk. ${ }^{15}$ According to the European Guideline on the Management of C. trachomatis Infections, the indication for laboratory tests for C. trachomatis is the presence of risk factor(s) and/or other sexually transmitted infections (STIs), i.e., age $<25$ years, new sexual contact in the last year or more than 1 partner in the last year. ${ }^{13}$ Laboratory tests are recommended for men aged $<40$ years with symptoms of acute epididymoorchitis and/or risk factors for STI, and for women with cervical or vaginal discharge with risk factors for STI, acute pain and/or PID symptoms. In both sexes, testing should be performed in all cases of rectal inflammation/colitis due to the risk of STIs and conjunctivitis; and in neonates, in cases of purulent conjunctivitis or atypical interstitial pneumonia. A separate group of patients referred for laboratory testing are those diagnosed with other STIs or having sexual contacts with STI or PID individuals, after pregnancy termination, or following any intrauterine interventions or manipulations. ${ }^{16}$

The aims of this study was to examine healthy women for C. trachomatis without symptoms from the reproductive system, to assess the frequency of asymptomatic infections, to detect GBS in the cervical canal, to demonstrate differences in drug susceptibility, and to determine the serotype of $S$. agalactiae strains and correlations among the bacterial strains present in the cervical canal.

\section{Material and methods}

All the procedures involving human participants were performed in accordance with the ethical standards of Wroclaw Medical University (Poland) and with the 1964 Helsinki declaration and its later amendments. The study protocol was accepted by the Ethics Committee of Wroclaw Medical University.

Cervical specimens were collected by gynecologists during prophylactic examinations. Swabs were taken from 315 women aged 18-32 years without previous genital symptoms from chlamydial or streptococcal infections. The mean age of the patients was $24.86 \pm 3.15$ years. Information on the number of partners in the previous year and the frequency of intercourse per week was collected from the patients during interviews. The women declared an average of 1.14 sexual partners in the previous 24 months and 2.05 sexual contacts per week. For chlamydia testing, we used a commercial DNA isolation kit and a C. trachomatis PCR kit (both from GeneProof a.s., Brno, Czech Republic) which allow simultaneous detection of a conservative region encoding 16S rRNA and a conservative region of a cryptic DNA plasmid, including deletion mutation in the cryptic plasmid (the Swedish variant). To detect GBS, specimens were tested using standard culturing methods. The cultures obtained were used to isolate small grey smooth colonies with $\beta$-hemolysis. The identification process included Gram-staining, and microscopic assessment of bacterial cells and colony purity. The Lancefield serological grouping was performed using a commercial streptococcal grouping kit (Oxoid Ltd., Basingstoke, UK). The culture properties of the collected $S$. agalactiae strains were tested using Columbia agar with 5\% sheep blood (Graso Biotech, Starogard Gdański, Poland) and selected media: either CHROMagar Strep B (Graso Biotech) or Granada agar/Columbia CNA $+5 \%$ sheep blood, (bioMérieux, Warszawa, Poland). Streptococcus agalactiae strains were identified and tested for their susceptibility to antibiotics (benzylpenicillin $1 \mathrm{IU}$, erythromycin $15 \mu \mathrm{g}$, clindamycin $2 \mu \mathrm{g}$; medium: Mueller-Hinton fastidious agar (MH-F); inoculum: 0.5 McFarland; incubation conditions: $5 \% \mathrm{CO}_{2}, 36^{\circ} \mathrm{C}, 18 \pm 2 \mathrm{~h}$ ). This 
methodology was recommended by the European Committee on Antimicrobial Susceptibility Testing (EUCAST). The isolates were serotyped using the Immulex ${ }^{\mathrm{TM}}$ StrepB kit (SSI Diagnostica, Hillerød, Denmark) for serotypes Ia, Ib, II, III, IV, V, VI, and VII. ${ }^{17}$

\section{Results}

Out of the 315 women involved in the study, 10 (3.2\%) were $C$. trachomatis-positive. Using traditional methods of microscopy, culture and serology, 42 strains obtained from patients (13.3\% of the subjects) were identified as $S$. agalactiae and further analyzed. The most common serotypes identified were II (in 18 out of the 42 , or $42.9 \%$ ), $\mathrm{V}(11 / 42,26.2 \%)$ and III $(10 / 42,23.8 \%)$. The less common serotypes found were VII $(2 / 42,4.8 \%)$ and Ib $(1 / 42,2.4 \%)$, with no Ia, IV or VII serotypes found. All the strains were susceptible to benzylpenicillin. The distribution of resistance-phenotypes among the GBS serotypes isolated from women without symptoms of infection is presented in Table 1.

Table 1. Distribution of resistance phenotypes among GBS serotypes isolated from women without symptoms of infection

\begin{tabular}{|l|c|}
\hline \multicolumn{1}{|c|}{ Serotype, $n$} & Resistant (\%) \\
\hline $\mathrm{Ib}(1)$ & $\mathrm{E}(100)$ \\
\hline$\|(18)$ & $\mathrm{E}(22.2), \mathrm{DA}(22.2)$ \\
\hline $\mathrm{III}(10)$ & $\mathrm{E}(10.0), \mathrm{DA}(10.0)$ \\
\hline $\mathrm{V}(11)$ & $\mathrm{E}(54.5), \mathrm{DA}(27.3)$ \\
\hline $\mathrm{VII}(2)$ & $\mathrm{E}(0), \mathrm{DA}(0)$ \\
\hline
\end{tabular}

Resistance phenotype: E - erythromycin; DA - clindamycin.

\section{Statistical analysis}

We found no correlation between positive results for C. trachomatis and GBS; the value of the $\chi^{2}$ test with the Yates's correction is $\chi^{2}(1)=0.248, p=0.875$. This result confirms the significance value of the asymptotic odds ratio $(\mathrm{OR})(\mathrm{p}=0.75)$ indicating that there is no relationship between the variables. Among the patients with positive results for $C$. trachomatis, $10 \%$ were also positive for GBS. In the GBS-positive study group, $2.4 \%$ were also positive for C. trachomatis.

\section{Discussion}

Infections caused by GBS are commonly detected in the vagina. In our study, Streptococcus were isolated in smears from the cervical canal. The GBS colonization was confirmed in 42 patients (13.3\%) and isolates were identified as $S$. agalactiae. The GBS colonization is transient in nature, and little is known about the host and bacterial factors controlling GBS persistence. Patras et al. used human cervical and vaginal epithelial cells in the mouse model of GBS vaginal colonization to characterize key host factors responsible for GBS colonization. ${ }^{3}$ The authors identified GBS strains that persisted for more than a month in the murine vagina, while other strains were more easily cleared. Moreover, they demonstrated that the persistent strain more readily invades cervical cells compared to vaginal cells, suggesting that GBS may potentially use the cervix as a reservoir to establish long-term colonization. The authors noted that compared to serotype Ia, serotype III had increased adherence to vaginal cells, which confirms the previously noted increased vaginal epithelial adherence of serotype III strains over serotype Ia strains. Furthermore, they demonstrated that serotype $\mathrm{V}$ invades and/or survives within the cervical epithelium more readily than other serotypes, which may be beneficial in niche establishment and long-term cervical-vaginal persistence. ${ }^{3}$

Similar results were obtained by Sadeh et al. in a study involving non-pregnant women. They obtained 70 isolates from 413 patients (16.9\%). The most numerous group were serotypes III (50\%), II (27.1\%) and V (12.9\%). ${ }^{18}$

Newborns most commonly contact GBS from the mother's genital tract. The GBS is detected in the vagina and rectum of $10-30 \%$ of pregnant women. ${ }^{3}$ Based on their capsular polysaccharides, GBS isolates can be divided into 9 different serotypes. However, the distribution of serotypes varies according to geographic location. In the present study, the most common serotypes found in 315 women were II (18/42, 42.9\%), V (11/42, 26.2\%) and III (10/42, $23.8 \%)$. Less common serotypes included VII (2/42, 4.8\%) and Ib $(1 / 42,2.4 \%)$, whereas serotypes Ia, IV and VII were not found at all. Persson et al. reported that the serotype distribution of colonizing strains is similar to the distribution of invasive strains, but 2 studies have shown that the proportion of serotype III strains is higher among invasive strains than among colonizing strains. ${ }^{1}$ This indicates that serotype III strains may be more virulent than strains of other serotypes, which could be related to a failure to obtain an adequate serum antibody response to serotype III during colonization.

There are some unexplained geographical differences between the serotype distributions of colonizing GBS strains. The most striking is the high prevalence of serotypes VI and VIII in pregnant Japanese women, whereas these strains seem currently absent or rare in Europe and North America. ${ }^{8}$ Serotypes Ia, Ib, II, and III prevail in many parts of the world, but serotype $\mathrm{V}$ is the most frequently isolated in many countries. ${ }^{19}$

Clinical manifestations of GBS infection in adults are numerous and quite varied. Since Group B streptococci can colonize skin and mucosal surfaces and may be isolated from infected sites along with other virulent organisms, their role in pathogenesis has often been questioned. However, studies of invasive GBS infection in which microorganisms are isolated from normally sterile sites, such 
as blood or cerebrospinal fluid (CSF), provide direct evidence that Group B streptococci are the etiologic agents in many clinical syndromes. ${ }^{13}$ Types Ia, III and V are currently the most frequently isolated serotypes from adult patients in nearly equal proportions. ${ }^{13} \mathrm{We}$ found a prevalence of serotypes II, V and III, the presence of which in the cervix may act as a reservoir for invasive diseases, even if they escalate over time or in combination with another underlying disease. Special attention should be paid to serotype III GBS, which is considered extremely virulent. Tazi et al. examined GBS strains isolated from adults with invasive infections in France and found that such infections are more frequent among people aged $\geq 65$ years of age, as described in other European and US surveys, with serotypes Ia, III and V accounting for $72 \%$ of all examined strains. ${ }^{20}$

In 2009, Brzychczy-Włoch et al. reported a different distribution of serotypes in pregnant women colonized by GBS: Those authors found that serotype III was predominant (35\%), followed by serotypes Ia (20\%), V (17\%) and II (15\%). They demonstrated that pregnancy hormones and changes in the reproductive system significantly affect GBS colonization and the distribution of predominant serotypes in pregnant women. ${ }^{21}$ Wolny-Koładka examined 250 S. agalactiae strains isolated from female reproductive tracts and found that the predominant serotypes were III (54\%) and V (12\%), followed by the Ia serotype (17\%), which was not found in our study. ${ }^{22}$ Epidemiological data from European countries demonstrate that Ia, II, III, and $\mathrm{V}$ are the most frequently isolated serotypes in European countries. ${ }^{1,6,7,21}$ Except for Ia, all these serotypes were confirmed in our study.

In our study, $71.4 \%$ of the strains were susceptible to erythromycin, $81.0 \%$ were susceptible to clindamycin, and the others $(19.0 \%)$ were clindamycin-resistant. We found 7 strains (16.7\%) that were simultaneously resistant to erythromycin and clindamycin. Garland et al. reported that in many countries, erythromycin resistance ranges from about $3 \%$ in Japan and Australia to $54 \%$ in the USA. ${ }^{23}$

Out of the 7 strains simultaneously resistant to erythromycin and clindamycin in our study, we found 4 strains with serotype II and 3 strains with serotype V. Strain with serotypes Ib was resistant to erythromycin. Jannati et al. conducted research in Iran to determine the serotype distribution and antibiotic resistance of 56 GBS strains. ${ }^{19}$ Their results showed that all the isolates were susceptible to penicillin. Only 3 isolates (5.3\%) were intermediate to erythromycin (serotypes II, V and 1 unidentified); the remaining isolates were susceptible to erythromycin. Only 2 isolates (3.5\%) were resistant to clindamycin (serotypes II and III); 2 (3.5\%) were intermediate; the rest were susceptible to clindamycin. ${ }^{19}$ Therefore, in our study and in the Iranian study, serotypes V, II and III were resistant to both erythromycin and clindamycin. Tazi et al. reported that erythromycin resistance has increased since 2007, reaching $35.24 \%$ in 2010; in their study, erythromycin resistance was prevalent in serotype $\mathrm{V}^{20}$
Because women who suffer from C. trachomatis infection may have no clinical symptoms, periodic or prophylactic health examinations can help detect and confirm chlamydial infection. In our study, PCR tests showed that $3.2 \%$ of the participants were Chlamydia-positive. According to the literature, in Europe, the level of C. trachomatis infections among women ranges between $3 \%$ and $5.3 \%,{ }^{13,16}$ which is consistent with the results of our research. It is also estimated that 70-95\% of infected women do not experience any symptoms in the genital system, which leads to uncontrolled spread of this microorganism. ${ }^{13,16}$ Undiagnosed and untreated patients are potential reservoirs of this bacterium. ${ }^{11}$ Detecting $C$. trachomatis infection before or during early pregnancy helps to avoid complications related to premature rupture of membranes, preterm birth and neonatal infections. ${ }^{13,16}$ In Germany, Dudareva-Vizule et al. analyzed the results for C. trachomatis obtained between 2008 and 2014, and found that $3.9 \%$ of the women were infected with this microorganism. ${ }^{24}$ In the age group $<25$ years, $26.9 \%$ of the women underwent screening tests. The highest percentages of positive results were found in women aged 15-19 years (5\%) and 20-24 years (4.9\%). Most of the women who underwent the tests were pregnant (41.9\%); these were preventive check-ups. $^{24}$

In 2008-2013, Bianchi et al. used a nested polymerase chain reaction (PCR) assay to detect $C$. trachomatis cryptic plasmid in cervical smears from women who had not reported any clinical symptoms of $C$. trachomatis infection. ${ }^{25}$ The authors detected $C$. trachomatis infection in $4.4 \%$ of the participants, with the highest percentage of infection recorded in women aged $20-21$ years $(5.5 \%)$ and the lowest in women aged $22-23$ years (3.5\%). The differences between infection rates in different age groups were not significant. ${ }^{25}$ The frequency of detecting chlamydial infections depends on the region and diagnostic methods. Genetic PCR tests are the reference method, but due to their limitations, immunofluorescence or enzymelinked immunosorbent assay (ELISA) assays are more often used. ${ }^{9}$ Arsić et al. obtained a significantly higher percentage of positive results in the Balkans than the published data by other authors indicated. ${ }^{26}$ In 1 center, C. trachomatis ELISA assays were positive in $7.1 \%$ of the patients (i.e., 100/1400); in Skopje, where direct fluorescent antibody (DFA) tests were used, Chlamydia antigens were detected in $6.8 \%$ of the women $(120 / 718) .{ }^{26}$ In our own earlier study (2012-2013), we examined investigated the presence of C. trachomatis in cervical swabs among women aged 1830 using direct immunofluorescence (DIF) and reported that $4 / 109$ patients (3.7\%) were Chlamydia-positive, ${ }^{14}$ which is very close to our current results obtained with PCR.

Chlamydia-positive sexual partners are at high risk of transmitting this pathogen through sexual contact. Therefore, both sexual partners should be examined for infection and treated at the same time. Berntsson et al. examined 99 women with partners who had tested positive 
for C. trachomatis. ${ }^{12}$ Using genetic methods, they obtained positive C. trachomatis results in 53 out of 99 women (53.5\%). Among the women infected with C. trachomatis, $50 \%$ did not declare any clinical symptoms, while the remaining $50 \%$ reported dysuria or vaginal discharge. ${ }^{12}$

Our study found no correlation between the presence of C. trachomatis and GBS colonization of the cervical canal. The coexistence of both microorganisms was found in only 1 patient. Neither Friedek et al. nor Honig et al. found this correlation, but they examined C. trachomatis in cervical swabs and streptococci in vaginal swabs. ${ }^{27,28}$

\section{Conclusions}

Early diagnosis, awareness of the need to look for deeply advanced infection and adequate antimicrobial therapy are essential elements of effective treatment for GBS infection. The high morbidity and mortality of invasive GBS infections has made the development of a multivalent conjugate polysaccharide vaccine a major focus for research. Awareness of the distribution of and changes in GBS serotypes in different populations is important. Continued surveillance of invasive GBS disease in adults and genetic characterization of the isolated strains are essential, as they may impact the use of antibiotics and vaccine design. In our study, the distribution of GBS serotypes was similar to results from other European countries and confirms the geographical variability of occurrence. Further research is needed to confirm the risk factors for GBS infection. Penicillin is used for GBS prophylaxis, and erythromycin and clindamycin remain suitable alternatives for women with $\beta$-lactam antibiotic allergies. Unfortunately, the number of strains resistant to macrolides and lincosamides is growing, so it is necessary to monitor the sensitivity of isolates of GBS. In our study, the incidence of C. trachomatis infections and GBS colonization is similar to other European countries. We found no positive correlation between the presence of GBS and C. trachomatis in the cervical canal. We did not find any correlation between the presence of $C$. trachomatis and the number of sexual partners or sexual contacts, because the percentage of positive results was too low.

\section{ORCID iDs}

Magdalena Frej-Mądrzak (1) https://orcid.org/0000-0002-8138-2586 Agnieszka Jama-Kmiecik (D) https://orcid.org/0000-0001-6514-8629 Jolanta Sarowska (D) https://orcid.org/0000-0001-9710-2721 Dorota Teryks-Wołyniec (D) https://orcid.org/0000-0003-0488-3579 Anna Gryboś (D) https://orcid.org/0000-0002-7456-4373 Marian Gryboś (D) https://orcid.org/0000-0003-0216-8088 Irena Choroszy-Król (D) https://orcid.org/0000-0002-5006-4059

\section{References}

1. Persson E, Berg S, Trollfors B, et al. Serotypes and clinical manifestations of invasive Group B streptococcal infections in western Sweden 1998-2001. Clin Microbiol Infect. 2004;10:791-796. doi:10.1111/j.14690691.2004.00931.x
2. Shabayek S, Spellerberg B. Group B streptococcal colonization, molecular characteristics, and epidemiology. Front Microbiol. 2018,9:437. doi:10.3389/fmicb.2018.00437

3. Patras KA, Rösler B, Thoman ML, Doran KS. Characterization of host immunity during persistent vaginal colonization by Group B Streptococcus. Mucosal Immunol. 2015;8(6):1339-1348. doi:10.1038/mi.2015.23

4. Lyhs U, Kulkas L, Katholm J, et al. Streptococcus agalactiae serotype IV in humans and cattle, Northern Europe. Emerg Infect Dis. 2016;22(12): 2097-2103. https://dx.doi.org/10.3201/eid2212.151447

5. Farley MM. Group B streptococcal disease in nonpregnant adults. Clin Infect Dis. 2001;33:556-561.

6. Wolny K, Gołda-Matuszak E. Streptococcus agalactiae (GBS): The characteristic of isolated strains from productive women's vagina. Med Dośw Mikrobiol. 2010;62:141-151.

7. Kierzkowska M, Majewska A, Kądzielska J, et al. Evaluation of bacterial flora found in the the cervix of pregnant women. Perinatologia, Neonatologia i Ginekologia. 2012;5(1):26-29.

8. Meyer T. Diagnostic procedures to detect Chlamydia trachomatis infections. Microorganisms. 2016;4(3):25. doi:10.3390/microorganisms 4030025

9. European Centre for Disease Prevention and Control. Chlamydia infection. In: ECDC Annual Epidemiological Report for 2017. Stockholm, Sweden: ECDC; 2019

10. Storey $C$, Chopra I. Affinities of $\beta$-lactams for penicillin binding proteins of Chlamydia trachomatis and their antichlamydial activities. Antimicrob Agents Chemother. 2001;45(1):303-305. doi:10.1128/AAC. 45.1.303-305.2001

11. Frej-Mądrzak M, Gryboś A, Gryboś M, et al. PCR diagnostics of Chlamydia trachomatis in asymptomatic infection by women. Ginekol Pol. 2018;89(3):115-119. doi:10.5603/GP.a2018.0020

12. Berntsson M, Tunbäck P. Clinical and microscopic signs of cervicitis and urethritis: Correlation with Chlamydia trachomatis infection in female STI patients. Acta Derm Venereol. 2013;93:230-233. doi:10. 2340/00015555-1536

13. Lanjouw E, Ouburg S, de Vries HJ, Stary A, Radcliffe K, Unemo M. 2015 European guideline on the management of Chlamydia trachomatis infections. Int J STD AIDS. 2016;27(5):333-348. doi:10.1177/0956 462415618837

14. Frej-Mądrzak M, Teryks-Wołyniec D, Jama-Kmiecik A, Sarowska J, Gościniak G, Choroszy-Król I. Chlamydia trachomatis infections of the genitourinary tract and conjunctiva in adults and children in 20122013. Fam Med Primary Care Rev. 2014;16(3):225-227.

15. Polish Gynecological Society recommendations regarding Chlamydia trachomatis infections in obstetrics and gynecology. Ginekol Pol. 2007;78:574-575.

16. European Centre for Disease Prevention and Control. Guidance on Chlamydia control in Europe - 2015. Stockholm, Sweden: ECDC; 2016. doi:10.2900/667703

17. The European Committee on Antimicrobial Susceptibility Testing. Breakpoint tables for interpretation of MICs and zone diameters. Version 9.0, 2019. http://www.eucast.org.

18. Sadeh M, Firouzi R, Derakhshandeh A, Khalili MB, Kong F, Kudinha T. Molecular characterization of Streptococcus agalactiae isolates from pregnant and non-pregnant women at Yazd University Hospital, Iran. Jundishapur J Microbiol. 2016;9(2):e30412. doi:10.5812/jjm.30412

19. Jannati E, Roshani M, Arzanlou M, Habibzadeh S, Rahimi G, Shapuri R. Capsular serotype and antibiotic resistance of group B streptococci isolated from pregnant women in Ardabil, Iran. Iran J Microbiol. 2012;4(3):130-135.

20. Tazi A, Morand PC, Reglier-Poupet $\mathrm{H}$, et al. Invasive group B streptococcal infections in adults, France (2007-2010). Clin Microbiol Infect. 2011;17(10):1587-1589. doi:10.1111/j.1469-0691.2011.03628.x

21. Brzychczy-Włoch M, Gosiewski T, Bodaszewska M, Pabian W, Bulanda M, Heczko PB. Analysis of serotypes distribution of Group B streptococci origin from pregnant carriage using multiplex PCR [in Polish]. Med Dośw Mikrobiol. 2009;61(4):293-299.

22. Wolny-Koładka K. Evaluation of ITS-PCR and PCR MP techniques for Streptococcus agalactiae genetic differentiation [in Polish]. Med Dośw Mikrobiol. 2014;66(3-4):149-160.

23. Garland SM, Cottrill E, Markowski L, et al. Antimicrobial resistance in Group B streptococcus: The Australian experience. J Med Microbiol. 2011;60(Pt 2):230-235. doi:10.1099/jmm.0.022616-0 
24. Dudareva-Vizule S, Haar K, Sailer A, et al; Chlamydia trachomatis Laboratory Sentinel Team. Establishment of a voluntary electronic Chlamydia trachomatis laboratory surveillance system in Germany, 2008 to 2014. Euro Surveill. 2017;22(6):30459.

25. Bianchi S, Frati ER, Canuti M, et al. Molecular epidemiology and genotyping of Chlamydia trachomatis infection in a cohort of young asymptomatic sexually active women (18-25 years) in Milan, Italy. J Prev Med Hyg. 2016;57(3):E128-E134.
26. Arsić D, Milovanović DR, Ferati AB, et al. Monitoring of Chlamydia trachomatis genitourinary infection in women: Analytical comparative study using public health records from two Balkan countries. Cent Eur J Public Health. 2016;24(1):16-21. doi:10.21101/cejph.a4088

27. Friedek $D$, Ekiel $A$, Romanik $M$, et al. Co-occurrence of urogenital mycoplasmas and Group B streptococci with chlamydial cervicitis. Pol J Microbiol. 2005;54(3):253-255.

28. Honig E, Mouton JW, van der Meijden WI. The epidemiology of vaginal colonisation with Group B streptococci in a sexually transmitted disease clinic. Eur J Obstet Gynecol Reprod Biol. 2002;105(2):177-180. 